\title{
ORTHODONTIC ADHESIVE SYSTEMS - OVER HALF A CENTURY OF RESEARCH AND EXPERIENCE
}

\author{
Dorota Kuśmierczyk', Konrad Małkiewicz² \\ 'Department of Orthodontics, Medical University of Warsaw, Poland \\ 2Department of Orthodontics, Medical University of Lodz, Poland
}

\begin{abstract}
Nowadays, during orthodontic treatment, brackets of fixed appliances are attached directly to tooth enamel. Research on development and improvement of orthodontic adhesives has been going on for over 50 years and is progressing in many directions. As a result, new solutions are introduced into clinical practice to improve functionality of adhesive systems being applied. Demands set for an adhesive system dedicated to orthodontic use, are primarily: ability to transfer orthodontic forces onto teeth in three spatial dimensions, resistance to masticatory forces, resistance to oral environment, ability to accurately position brackets and maintain them throughout treatment, possibility of detaching brackets without causing damage to tooth enamel and biocompatibility. Products available on the market do not constitute a homogeneous group in terms of both chemical structure and functional features. This paper presents the history of development of orthodontic adhesive systems and their features in relation to clinical use. Attention was also paid to directions of evolution of the aforementioned group of materials aimed at improving their performance in certain aspects of medical practice. Despite the ongoing research and development work, no orthodontic adhesive system that meets all the requirements set for "ideal material" has been developed to date. Orthodontic adhesives introduced into the market require assessment of their actual clinical usability and safety in treatment of patients.
\end{abstract}

KEY WORDS: polymers, composite resins, orthodontic adhesives, fixed appliances.

J Stoma 2019; 72, 4: 179-183

DOI: https://doi.org/10.5114/jos.2019.91236

\section{INTRODUCTION}

The need to develop materials capable of creating strong and lasting bonds with tooth tissues was noted already in the 1950s. Development of modern adhesive dentistry was possible thanks to a discovery made in 1955 by M.G. Buonocuore [9], who observed that the bond between acrylic resin and an enamel surface significantly strengthened if the latter was previously treated with $85 \%$ orthophosphoric acid, compared to a bond of material with chemically untreated enamel. This observation initiated development of a tooth enamel etching procedure in order to strengthen the adhesion of dental materials, including orthodontic adhesives, to its surface [31].

The demands for an adhesive system dedicated to orthodontic use, such as ability to transfer orthodontic forces onto teeth in three spatial dimensions, resistance to masticatory forces, resistance to the oral environment, ability to accurately position brackets and maintain them throughout treatment and possibility of detaching brackets without causing damage to tooth enamel [32], for many years could not be met to a sufficient degree by materials developed in individual research centers [25]. The advantages of direct attachment of orthodontic brack-

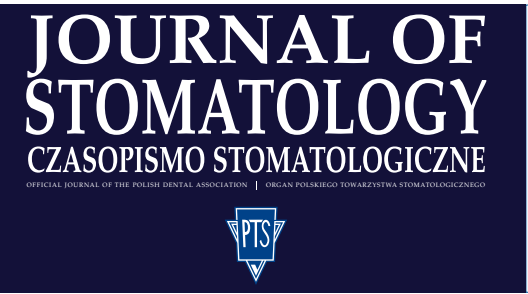

ADDRESS FOR CORRESPONDENCE: Dorota Kuśmierczyk, Department of Orthodontics, Medical University of Warsaw, 6 Stanisława Binieckiego St., 02-097 Warsaw, Poland, e-mail: dkusmierczyk@wum.edu.pl

RECEIVED: 31.07.2019 • ACCEPTED: 01.12.2019 • PUBLISHED: 12.12.2019 
ets to tooth enamel compared to treatment techniques using appliances based on bands placed on all teeth were already recognized in the 1970s. These include: improved patient's comfort by eliminating the stages of tooth separation and laborious adjustment of the bands, facilitating attachment of brackets to unusually shaped teeth or to not completely erupted teeth, limiting the risk of mechanical irritation to gingiva by the appliance's components, improved ability to maintain oral hygiene, reduced risk of enamel decalcification, lack of necessity to close spaces remaining after removal of bands from teeth, improved aesthetics of dentition during treatment and shortened dentist's working time [25].

The aim of the study is to present the history of development and to discuss the characteristics of orthodontic adhesive systems used in the past and nowadays.

\section{EVOLUTION OF ORTHODONTIC ADHESIVE SYSTEMS}

\section{CHEMICALLY CURED ORTHODONTIC ADHESIVES}

Among the materials initially tested for their use in direct fixing of orthodontic brackets onto the enamel surface of teeth, the majority were products of polymerization or copolymerization of methacrylic acid derivatives. Research conducted at the turn of the 1960s and 1970s by Newman [32] and Miura et al. [29] confirmed that the products were characterized by ease of mixing and application of adhesive, relatively high chemical stability as well as desired physical parameters. The first adhesive resins were packaged in the form of powdered polymethacrylates and liquid methacrylate monomers. The disadvantage of these systems was, among other things, long time of initial polymerization, which equaled 30 minutes [7].

In the 1960s, Rafael Bowen [8] patented material for filling cavities, which included

2,2-bis-[4-(2-hydroxy-3-methacryloxy-propoxy)phenyl] propane (Bis-GMA) as the main component of the organic phase and silica used as a filler. This formula was so successful that the polymer composite matrices based on Bis-GMA resin dominated the composition of cavity filling materials and orthodontic adhesives for many years [35]. In 1972 composite materials based on Bis-GMA resin were introduced, supplied in the form of two pastes requiring mixing: Adaptic (Johnson and Johnson, USA) and Concise (3M Unitek, USA) [50]. Originally used as filling materials, they were also applied in orthodontics $[6,20]$. In 1977 , the orthodontic adhesive system Concise Ortho Adhesive (3M Unitek, USA) was introduced for commercial use with a slightly modified composition compared to the restorative material [46]. In 1975, Gange [20] developed a chemically cured orthodontic adhesive for Lee Pharmaceuticals (USA) in a formula that did not require mixing. Polymerization of a single paste was initiated by contacting the material with surfaces of etched enamel coated with liquid catalyst and the base of the bracket. The effectiveness of polymerization and the strength of bonding the bracket with the enamel surface depended on the degree of matching between the bracket base and the shape of the enamel surface, conditioning access of the catalyst to the material mass.

\section{LIGHT-CURED ORTHODONTIC ADHESIVES}

Inconveniences associated with the use of chemically cured bonding systems led to research on orthodontic adhesives, whose polymerization process could be controlled and initiated depending on clinical indications. In the 1970s, the first adhesives polymerized with ultraviolet (UV) radiation with a wavelength of $280 \mathrm{~nm}$ [12] were presented. The adverse effect of UV radiation on the skin and eyes as well as recorded cases of soft tissue burns resulted in withdrawal from that method of initiating the polymerization process [20].

At the turn of the 1970s and 1980s, the first reports appeared on the use of composite materials in orthodontics, whose polymerization was initiated by visible light with a wavelength from 440 to $480 \mathrm{~nm}$ [20,44, 50]. Studies conducted in the following years confirmed that light-cured orthodontic adhesives offer longer working time needed for precise positioning of brackets on tooth surfaces, the moment of initiation of the polymerization process depending on the needs of the clinician, and high initial binding strength with a relatively low effect of oxygen inhibition on monomer crosslinking [17]. Thanks to these features, light-cured materials presently constitute the majority of orthodontic adhesive systems used by clinicians.

The disadvantage of the first light-cured orthodontic adhesives was the relatively long exposure period needed for polymerization, which at the same time did not guarantee full cross-linking of resin. The solution to this problem was supposed to be experimental adhesive systems that combined two ways of polymerization initiation: by visible light and by chemical reaction, the so-called dual cure cements. They were characterized by a limited period of plasticity, but they required a relatively short exposure time and ensured continuation of the monomer cross-linking process, catalyzed by chemical reaction [43]. An inheritor to the idea of double initiation of the polymerization process of orthodontic adhesive systems is a resin-reinforced glass ionomer cement, the Fuji Ortho LC resin (GC Corp., Japan), introduced in 1994 [42].

One of the adverse effects associated with the structure of the matrix of orthodontic adhesives based on composite materials is incomplete cross-linking during the polymerization process, leading to susceptibility of materials to degradation $[16,41]$. The percentage of double bonds undergoing saturation during the crosslinking process in the polymer is described as the de- 
gree of material conversion (\% DC). It depends on the composition of the material, and, in the case of materials cured with visible light, the process of creating an organic matrix is significantly impacted by radiation intensity, exposure time, distance from the light source to the material or thickness of the layer of polymerized adhesive. To date, no dental material has been produced whose matrix undergoes a $100 \%$ conversion to form a stable, spatial network. Data published in the literature indicate that only about $25.5-89.5 \%$ of monomer double bonds are involved in the polymerization of orthodontic adhesives $[17,21,38]$.

The next aim for teams of researchers were further modifications of orthodontic adhesives that would facilitate their application and guarantee even more beneficial usability.

\section{MODIFICATION OF FUNCTIONAL FEATURES OF ORTHODONTIC ADHESIVE SYSTEMS IN SELECTED ASPECTS OF CLINICAL PRACTICE}

One of the problems faced by clinicians is the excess of adhesive escaping from under the bracket base, contributing to increase of dental plaque retention and causing formation of unattractive discolorations around brackets. The solution turned out to be introduction of adhesive systems characterized by a contrasting color before cross-linking, which facilitates removal of their excess prior to bonding with brackets [20]. At the beginning of the $21^{\text {st }}$ century, numerous light-cured adhesive systems appeared, using the described solution, among which the most popular are Transbond Plus (3M Unitek, USA), and Grengloo and Blugloo (Ormco Corporation, USA) [15, 20].

Another challenge was development of adhesive systems resistant to moisture and contamination of the treatment field with organic fluids. It is not always possible to keep the treatment field dry, especially in cases of fastening brackets to surgically exposed retained teeth. At the beginning of the 1990s, cyanoacrylate adhesives resistant to moisture were introduced into orthodontic practice [24]. An example is the SmartBond chemically cured resin (Gestenco International, Sweden), based on ethyl cyanoacrylate. Numerous studies have confirmed the usability of the adhesive in conditions of tooth surface contamination with saliva and blood [34]. A light-cured version of this system, Smartbond LC, was also introduced onto the market [11].

Another method to solve the above-mentioned problem was development of hydrophilic orthodontic adhesive resins in the late 1990s, such as: Ortho Solo (Ormco, USA), Transbond MIP Moisture Insensitive Primer (3M Unitek, USA) or Assure (Reliance Orthodontics, USA) [20] The ethyl alcohol or acetone included in their composition is designed to displace water molecules from surfaces of etched enamel [48]. The published research results indicate that under conditions of inadequate moisture control or contamination of the tooth surface with blood or saliva, hydrophilic adhesive resins demonstrate clinically acceptable binding strength to the enamel surface and are the products of choice $[10,28]$. At the end of 2000, a self-etching adhesive system developed for the needs of orthodontics Transbond Plus SEP (self etch primer) (3M Unitek, USA), which did not require an absolutely dry treatment field, was introduced to the market. Studies confirm that the above-mentioned resin, not requiring a separate enamel etching procedure, secures a satisfactory bond between the bracket and enamel [10].

In order to shorten the time and to simplify the procedure of fixing orthodontic brackets, adhesive pre-coated brackets bases (APC) were introduced to the market. The advantages of APC systems, compared to conventional brackets requiring application of adhesive during the procedure, include: constant quality and quantity of adhesive on the bracket base, less excess adhesive resin around brackets, maintaining a high degree of asepsis of orthodontic materials and better control of use of purchased materials [13]. Comparative studies of APC systems and conventional orthodontic adhesives conducted in subsequent years, however, did not show a clear advantage of this solution in the context of bond strength between brackets and tooth tissues, the number of long-term clinical failures or dentist's saved time [1]. In 2013, the APC Flash-Free system of brackets (3M Unitek, USA), whose bases are covered with a type of mat impregnated with low viscosity adhesive resin, was launched for sale. The solution eliminates the phenomenon of excess adhesive flowing out of the bracket base during its positioning. In a clinical study published in 2018, Grundheid et al. [22] assessed the loss rate of brackets in the APC Flash-Free system, which turned out to be comparable to the rates obtained when assessing conventional orthodontic adhesives. Also, the time needed to fix a permanent appliance in the APC FlashFree system was noticeably shorter.

Attempts were made to reduce enamel demineralization caused by accumulation of dental plaque around components of fixed appliances, by introducing fluorideexchanging resin (FER), fluoride releasing bonding adhesives [33, 40, 45], e.g. Light Bond (Reliance Orthodontic Products, USA) or Transbond Plus Color Change Adhesive (3M Unitek, USA). Although the results of laboratory experiments confirmed the release of fluoride ions from composite material-based orthodontic adhesives [33], the results of clinical trials were equivocal [14, 40, 45].

Nowadays, research is conducted on new formulas of orthodontic adhesives enriched with compounds of proven antibacterial properties (e.g. catechins, benzalkonium chloride, chlorhexidine, triclosan, zinc oxide, silver nanoparticles, titanium dioxide, quaternary ammonium compounds). Results of in vitro tests, however, are inconclusive as far as sufficiently confirming their clinical usefulness $[3,4]$. Another solution proposed by 
research teams is introduction of bioactive glass (BAG) into the composition of orthodontic adhesives. The results of published studies indicate that dental materials enriched with BAG release calcium, phosphate and fluoride ions to the external environment, which - in addition to buffering the acidic oral environment - may contribute to remineralization of the previously damaged enamel structure [2].

One of the essential elements of orthodontic treatment with permanent appliances fixed with the use of adhesive technique is the procedure of removal of brackets and adhesive residues, performed after the treatment has been completed or, in the case of incorrect bracket positioning, also during treatment. High bonding power characterizing modern adhesive systems is associated with the risk of enamel surface damage as well as with pain and discomfort reported by patients during the procedure. The solution to this problem may be introduction of microcapsules with high thermal expansion into the structure of adhesive material. Increasing the temperature of the bracket and adhesive to an appropriate, safe level (e.g. with a laser beam) is supposed to result in a significant increase in the volume of the said particles, cracking of the adhesive material and, consequently, in a decrease in binding strength of the adhesive system [5].

\section{CHEMICAL STABILITY OF ORTHODONTIC ADHESIVE SYSTEMS IN THE ORAL ENVIRONMENT}

Contemporary orthodontic adhesives are a heterogeneous group of materials that differ in chemical composition, strength parameters and functional properties. These materials, which are introduced into the oral cavity during orthodontic treatment, are subject to the influence of this environment. The complex oral ecosystem is characterized by high humidity, significant temperature fluctuations, $\mathrm{pH}$ variability, electrochemical and enzymatic activity as well as the influence of masticatory forces. Unfavorable environmental conditions intensify degradation of materials used in dentistry, including orthodontics [49]. Many research teams focus their activities not only on assessing the usability of dental adhesive materials, but also on validating the stability of their chemical structure $[30,36]$ in the context of potential biological effects [39] on the patient's body. It has been shown that potentially harmful substances may be released into the patient's body as a result of insufficient polymerization of dental adhesive systems [47], or following degradation and aging of materials [27]. Numerous studies have confirmed the cyto- and genotoxicity of monomers and oligomers used in production of dental composite materials [23]. In the literature there are relatively few publications evaluating the release of harmful biological components from orthodontic ad- hesive systems. However, some studies have confirmed that assessed materials release Bis-GMA, triethylene glycol dimethacrylate (TEGDMA) and bisphenol A (BPA) to the external environment $[18,21,26,30,38]$.

It has been shown that TEGDMA has the ability to stimulate the growth of cariogenic bacterial cultures [23] and to cause allergic reactions [37]. However, particular concern in the context of the use of materials during dental treatment is raised by reports of the paraestrogenic effect of both Bis-GMA resin and bisphenol A (BPA) [19].

The wide spectrum of the negative impact of these chemical compounds on the human body is currently the subject of research in many scientific centers across the world.

\section{CONCLUSIONS}

Contemporary orthodontic adhesive systems constitute a heterogeneous group of materials differing not only in chemical composition but also in strength parameters and functional properties. The complex oral ecosystem in which they are used promotes their degradation, which should also be considered in the context of the potential biological impact of released components on the patient's body.

Despite the development of material techniques, up to the present time no orthodontic adhesive system has been introduced that meets all functional demands of the "perfect material". It should also be remembered that both types of resins, those developed decades ago and new adhesive resins with a modified chemical formula, require assessment in terms of their safety for use on patients.

\section{CONFLICT OF INTEREST}

The authors declare no potential conflicts of interest with respect to the research, authorship, and/or publication of this article.

\section{References}

1. Alakttash AM, Fawzi M, Bearn D. Adhesive precoated bracket systems and operator coated bracket systems: is there any difference? A systematic review and meta-analysis. Angle Orthod 2019; 89: 495-504

2. Al-eesa NA, Johal A, Hill RG, Wong FSL. Fluoride containing bioactive glass composite for orthodontic adhesives - apatite formation properties. Dent Mater 2018; 34: 1127-1133.

3. de Almeida CM, da Rosa WLO, Meereis CTW, et al. Efficacy of antimicrobial agents incorporated in orthodontic bonding systems: a systematic review and meta-analysis. J Orthodont 2018; 45: 79-93.

4. Altmann ASP, Collares FM, Leitune VCB, Samuel SMW. The effect of antimicrobial agents on bond strength of orthodontic adhesives: a meta-analysis of in vitro studies. Orthodont Craniofac Res 2016; 19: 1-9.

5. Arima S, Namura Y, Tamura T, Shimizu N. Easy debonding of ceramic brackets bonded with a light-cured orthodontic adhesive containing microcapsules with a $\mathrm{CO}_{2}$ laser. Photomed Laser Surg 2018; 36: 162-168 
6. Årtun J, Zachrisson B. Improving the handling properties of a composite resin for direct bonding. Am J Orthod Dentofacial Orthop 1982; 81: 269-276.

7. Bishara SE, Khowassah MA, Oesterle LJ. Effect of humidity and temperature changes on orthodontic direct-bonding adhesive systems. J Dent Res 1975; 54: 751-758.

8. Bowen RL. Dental filling material comprising vinyl silane treated fused silica and a binder consisting of the reaction of bisphenol and glycidyl acrylate. US Patent No. 3,066,112, Nov 27, 1962.

9. Buonocore MG. A simple method of increasing the adhesion of acrylic filling materials to enamel surfaces. J Dent Res 1955; 34: 849-853.

10. Cacciafesta V, Sfondrini MF, De Angelis M, et al. Effect of water and saliva contamination on shear bond strength of brackets bonded with conventional, hydrophilic, and self-etching primers Am J Orthod Dentofacial Orthop 2003; 123: 633-640.

11. Cacciafesta V, Sfondrini M, Gatti S, Klersy C. Effect of water and saliva contamination on the shear bond strength of a new lightcured cyanoacrylate adhesive. Prog Orthod 2007; 8: 100-111.

12. Cohl ME, Green LJ, Eick JD. Bonding of clear plastic orthodontic brackets using an ultraviolet-sensitive adhesive. Am J Orthod 1972; 62: 400-411.

13. Cooper RB, Goss M, Hamula W. Direct bonding with light cured adhesive precoated brackets. J Clin Orthod 1992; 26: 477-479.

14. Cury JA, de Oliveira BH, Dos Santos AP, Tenuta LM. Are fluoride releasing dental materials clinically effective on caries control? Dent Mater 2016; 32: 323-333.

15. Ekhlassi S, English JD, Ontiveros JC, et al. Bond strength comparison of color-change adhesives for orthodontic bonding using a self-etching primer. Clin Cosmet Investig Dent 2011; 3: 39-44

16. Eliades G, Eliades T, Vavuranakis M. General aspects of biomaterial surface alterations following exposure to biologic fluids. In Dental Materials in Vivo, Aging and Related Phenomena. Chicago: Quintessence Publishing Co Inc.; 2003, pp. 3-20.

17. Eliades T, Eliades G, Brantley WA, Johnston WM. Polymerization efficiency of chemically cured and visible light-cured orthodontic adhesives: Degree of cure. Am J Orthod Dentofac Orthop 1995; 108: 294-301.

18. Eliades T, Voutsa D, Sifakakis I, Makou M, Katsaros Ch. Release of bisphenol A from light-cured adhesive bonded to lingual fixed retainers. Am J Orthod Dent Orthop 2011; 139: 192-195.

19. Eramo S, Urbani G, Sfasciotti GL, Brugnoletti O, Bossù M, Polimeni A Estrogenicity of bisphenol A released from sealants and composites: a review of the literature. Ann Stomatol (Roma) 2010; 1: 14-21.

20. Gange P. The evolution of bonding in orthodontics. Am J Orthod Dentofac Orthop 2015; 147 (4 Suppl): 56-63.

21. Gioka Ch, Bourauel Ch, Hiskia A, Kletsas D, Eliades T, Eliades G. Light-cured or chemically cured orthodontic adhesive resin? A selection based on the degree of cure, monomer leaching and cytotoxicity. Am J Orthod Dent Orthop 2005; 127: 413-419.

22. Grünheid T, Larson BE. Comparative assessment of bonding time and 1-year bracket survival using flash-free and conventional adhesives for orthodontic bracket bonding: A split-mouth randomized controlled clinical trial. Am J Orthod Dentofacial Orthop 2018; 154: 621-628.

23. Gupta S, Saxena P, Pant V, Pant A. Release and toxicity of dental resin composite. Toxicol Int 2012; 19: 225-234.

24. Kahl B, König A, Hilgers RD, Schwarze CW. Ethyl cyanoacrylate (Cyano-Veneer) as an orthodontic bracket adhesive. A comparative in-vitro study with Cyano-Veneer and a conventional orthodontic bracket adhesive. Fortschr Kieferorthop 1993; 54: 263-267.

25. Khowassah MA, Bishara SE, Francis TC, Henderson W. Effect of temperature and humidity on the adhesive strength of orthodontic direct bonding materials. J Dent Res 1975; 54: 146-151.

26. Kloukos D, Pandis N, Eliades T. Bisphenol-A and residual monomer leaching from orthodontic adhesive resins and polycarbonate brackets: a systematic review. Am J Orthod Dentofacial Orthop 2013; 143: S104-112.

27. Krüger J, Maletz R, Ottl P, Warkentin M. In vitro aging behavior of dental composites considering the influence of filler content, storage media and incubation time. PLoS One 2018; 13: e0195160.
28. Kula KS, Nash TD, Purk JH. Shear-peel bond strength of orthodontic primers in wet conditions. Orthod Craniofac Res 2003; 6: 96-100.

29. Miura F, Nakagawa K, Masuhara E. New direct bonding system for plastic brackets. Am J Orthod 1971; 59: 350-361.

30. Moreira MR, Matos LG, de Souza ID, et al. Bisphenol A release from orthodontic adhesives measured in vitro and in vivo with gas chromatography. Am J Orthod Dentofacial Orthop 2017; 151: 477-483.

31. Mulholland RD, de Shazer DO. The effect of acidic pretreatment solutions on the direct bonding of orthodontic brackets to enamel. Angle Orthod 1968; 38: 236-243.

32. Newman GV, Snyder WH, Wilson CE. Acrylic adhesives for bonding attachments to tooth surfaces. Angle Orthod 1968; 38: 12-18.

33. Øgaard B, Rezk-Lega F, Ruben J, Arends J. Cariostatic effect and fluoride release from a visible light-curing adhesive for bonding of orthodontic brackets. Am J Orthod Dentofacial Orthop 1992; 101: 303-307.

34. Öztoprak MO, Isik F, Sayınsu K et al. Effect of blood and saliva contamination on shear bond strength of brackets bonded with 4 adhesives. Am J Orthod Dentofacial Orthop 2007; 131: 238-242.

35. Papakonstantinou AE, Eliades T, Cellesi F, et al. Evaluation of UD MA's potential as a substitute for Bis-GMA in orthodontic adhesives. Dent Mater 2013; 29: 898-905.

36. Pelourde Ch, Bationo R, Boileau MJ, Colat-Parros J, Jordana F Monomer release from orthodontic retentions: an in vitro study. Am J Orthod Dentofacial Orthop 2018; 153: 248-254.

37. Peterson M, Wong P, Dickson S, Coop ChA. Allergic stomatitis from orthodontic adhesives. Mil Med 2017; 182: 1883-1885.

38. Purushothaman D, Kailasam V, Chtharanjam AB. Bisphenol A release from orthodontic adhesives and its correlation with the degree of conversion. Am J Orthod Dent Orthop 2015; 147: 29-36.

39. Rai AK, Ansari A, Gera A, Jain AK. Toxicological aspect of orthodontic bonding materials. Virtual Journal of Orthodontics [serial online] 2012; 9; http://www.vjo.it/.

40. Rogers S, Chadwick B, Treasure E. Fluoride-containing orthodontic adhesives and decalcification in patients with fixed appliances: a systematic review. Am J Orthod Dentofacial Orthop 2010; 138 390.e1-390.e8.

41. Santerre JP, Shajii L, Leung BW. Relation in dental composite formulations to their degradation and release of hydrolyzed polymeric resin derived product. Crit Rev Oral Biol Med 2001; 12: 136-151.

42. Silverman E, Cohen M, Demke RS, Silverman M. A new lightcured glass ionomer cement that bonds brackets to teeth without etching in the presence of saliva. Am J Orthod Dentofacial Orthop 1995; 108: 231-236.

43. Smith RT, Shivapuja PK. The evaluation of dual orthodontic bonding cement resin. Am J Orthod Dentofac Orthop 1993; 103: 448-451.

44. Tavas MA, Watts DC. Bonding of orthodontic brackets by transillumination of a light activated composite: an in vitro study. $\mathrm{Br}$ J Orthod 1979; 6: 207-208

45. Trimpeneers LM, Dermaut LR. A clinical evaluation of the effectiveness of a fluoride-releasing visible light-activated bonding system to reduce demineralization around orthodontic brackets. Am J Orthod Dentofacial Orthop 1996; 110: 218-222.

46. Wahl N. Orthodontics in 3 millennia. Chapter 16: late 20th-century fixed appliances. Am J Orthod Dentofacial Orthop 2008; 134: 827-830.

47. Walters NJ, Xia W, Salih V, Ashley PF, Young AM. Poly(propylene glycol) and urethane dimethacrylates improve conversion of dental composites and reveal complexity of cytocompatibility testing. Dent Mater 2016; 32: 264-277

48. Webster MJ, Nanda RS, Duncanson MG, et al. The effect of saliva on shear bond strengths of hydrophilic bonding systems. Am J Orthod Dentofacial Orthop 2001; 119: 54-58.

49. Wołowiec P, Chojnacka K, Loster BW, Mikulewicz M. Do dietary habits influence trace elements release from fixed orthodontic appliances? Biol Trace Elem Res 2017; 180: 214-222.

50. 100 years of orthodontic history. Am J Orthod Dentofacial Orthop 2015; 148: 875-876. 